ASTHMA

\title{
Relationship of airway wall thickening to an imbalance between matrix metalloproteinase- 9 and its inhibitor in asthma
}

\author{
H Matsumoto, A Niimi, M Takemura, T Ueda, M Minakuchi, R Tabuena, K Chin, T Mio, Y Ito, \\ S Muro, T Hirai, S Morita, S Fukuhara, M Mishima
}

Thorax 2005;60:277-281. doi: 10.1136/thx.2004.028936

\begin{abstract}
See end of article for authors' affiliations

....................

Correspondence to: Dr A Niimi, Department of Respiratory Medicine Graduate School of Medicine, Kyoto University, Sakyo-ku, Kyoto 606-8507, Japan; niimi@kuhp.kyoto-u.ac.jp
\end{abstract}

Received 21 May 2004 Accepted

21 December 2004

\begin{abstract}
Background: The balance between matrix metalloproteinase-9 (MMP-9) and tissue inhibitor of metalloproteinase-1 (TIMP-1) may be critical in extracellular matrix remodelling, a characteristic of asthmatic airways. An excess of TIMP-1 over MMP-9 has been associated with chronic airflow obstruction but the mechanisms underlying this association remain unknown. Recent computed tomographic (CT) studies indicate that airway wall thickening is associated with chronic airflow obstruction.

Methods: Sputum levels of MMP-9, TIMP-1, and their molar ratio were examined in 26 patients with stable asthma and their relationship with pulmonary function and airway wall thickness, assessed by a validated CT technique which measured wall area corrected by body surface area (WA/BSA), the ratio of WA to outer wall area (WA\%), and the absolute wall thickness corrected by $\sqrt{ }$ BSA of a segmental bronchus (T/ (BSA), was examined.

Results: Sputum MMP-9 levels were inversely correlated with WA\% and TIMP-1 levels were positively correlated with WA/BSA and T//BSA. The MMP-9/TIMP-1 molar ratio was inversely correlated with WA $\%$ and $T / \sqrt{ }$ BSA and positively correlated with post-bronchodilator values of mid-forced expiratory flow and maximum expiratory flow at the quartile of lung volume.

Conclusion: Excess TIMP-1 may have a pathogenetic role in airway wall thickening in asthmatic patients which may result in chronic airflow obstruction.
\end{abstract}

1 nflammation of the airway is a characteristic feature of asthma. Pathological repair of chronic inflammation may result in airway remodelling characterised by hypertrophy and hyperplasia of airway smooth muscle, submucosal gland hyperplasia, goblet cell hyperplasia, and vascular proliferation. Structural changes also include deposition of extracellular matrix (ECM), such as collagen and fibronectin, in the subepithelial basement membrane (SBM) or in the submucosa, around or within airway smooth muscle bundles, and possibly in the adventitia. ${ }^{12}$

Matrix metalloproteinases (MMPs), a family of zinc- and calcium-dependent enzymes, are responsible for the degradation of ECM. ${ }^{3}$ Among MMPs, MMP-9 is overexpressed in the serum and/or diseased organs of patients with several disorders characterised by tissue destruction, such as rheumatoid arthritis and pulmonary emphysema. ${ }^{45}$ MMP-9 is considered to be involved in the pathophysiology of these disorders, presumably by degrading ECM. In patients with asthma, MMP-9 is increased in sputum or bronchoalveolar lavage (BAL) fluid under stable conditions, ${ }^{67}$ during spontaneous exacerbations, ${ }^{8}$ and after local allergen challenge. ${ }^{9}$ MMP-9 has also been suggested to enhance the migration of inflammatory cells into the airways ${ }^{10}$ through ECM destruction. ${ }^{11}$

As a counterbalance, tissue inhibitor of metalloproteinase1 (TIMP-1) inhibits the enzymatic activity of MMP-9 by stoichiometric $1: 1$ binding. ${ }^{12}$ TIMP- 1 is considered to have fibrogenic properties resulting from inhibition of MMP-9 and promotion of cell growth of fibroblasts or myofibroblasts. ${ }^{13}$ In several fibrotic diseases, such as progressive systemic sclerosis $^{14}$ and idiopathic pulmonary fibrosis, ${ }^{15}$ TIMP- 1 is overexpressed and considered to accelerate fibrosis and ECM deposition. In the sputum or BAL fluid of patients with stable asthma, TIMP-1 is increased compared with healthy controls and is overproduced relative to MMP-9. ${ }^{616}$ Additionally, an excess of TIMP-1 over MMP-9 in the sputum or serum of asthmatic patients has been associated with chronic airflow obstruction. ${ }^{6}{ }^{17}$

Necroscopic studies indicate that airway wall thickening is involved in the pathophysiology of asthma. ${ }^{18}$ We have established a technique of helical computed tomography (CT) to quantify airway wall thickness which has shown that the airways of asthmatic patients are thickened in vivo. ${ }^{19} 20$ The degree of thickening may relate to the severity of disease and airflow obstruction. ${ }^{19-22}$ Chronic airflow obstruction associated with overproduction of TIMP-1 might therefore theoretically result from deposition of ECM and consequent thickening of the airway wall. ${ }^{17}$ We therefore investigated the relation of sputum levels of MMP-9 and TIMP-1 and their molar ratio with airway wall thickness as assessed by CT in patients with stable asthma.

\section{METHODS}

Subjects

Twenty six patients with stable asthma from our outpatient clinic were studied. Asthma was diagnosed according to the American Thoracic Society criteria. At the time of diagnosis, airway hyperresponsiveness was proven in 15 patients by a continuous inhalation methacholine challenge; ${ }^{23}$ the mean (range) Dmin, a marker of airway sensitivity, in these 15

Abbreviations: Ao, outer area of the bronchus; BSA, body surface area; $\mathrm{ECM}$, extracellular matrix; $\mathrm{FEF}_{25-75 \%}$, mid forced expiratory flow; $\mathrm{FEV}_{1}$, forced expiratory volume in 1 second; MMP-9, matrix metalloproteinase-9; SBM, subepithelial basement membrane; $T$, absolute airway thickness; TIMP-1, tissue inhibitor of metalloproteinase1; WA, airway wall area 
patients was 1.96 (0.01-9.53) units. Reversibility of airflow obstruction to $200 \mu \mathrm{g}$ salbutamol ( $12 \%$ and $200 \mathrm{ml}$ increase in forced expiratory volume in l second $\left.\left(\mathrm{FEV}_{1}\right)\right)$ was demonstrated in six patients (mean (SD) pre/post-bronchodilator $\mathrm{FEV}_{1} 2.36$ (0.85) l/2.81 (1.02) l), and the remaining five patients had peak expiratory flow (PEF) variability of more than $20 \%$. Patients were considered to have stable asthma if the disease had been fully controlled for at least 1 month. ${ }^{7}$ Twenty of the 26 patients had never smoked; the remaining six were ex-smokers who had ceased smoking at least 1 year before the study and had smoked a maximum of 10 pack-years. Emphysema was ruled out by CT images in all subjects including the six ex-smokers.

The study was approved by the ethics committee at our institution and written informed consent was obtained from all participants.

\section{Sputum induction and processing}

Sputum induction and processing were performed according to the methods of Pin ${ }^{24}$ with slight modification. ${ }^{25}$ Briefly, the subjects were premedicated with inhaled salbutamol $(200 \mu \mathrm{g})$ and then inhaled hypertonic (3\%) saline solution from an ultrasonic nebuliser (MU-32, Azwell Inc, Osaka, Japan) for 15 minutes. Adequate plugs of sputum were separated from saliva and first treated with $0.1 \%$ dithiothreitol (Sputasol, Oxoid Ltd, Hampshire, UK) followed by Dulbecco's phosphate buffered saline (PBS). After centrifugation, supernatants of sputum were stored at $-80^{\circ} \mathrm{C}$. Cell differentials were determined by counting at least 400 nonsquamous cells stained by the May-Grünwald-Giemsa method.

\section{Zymography}

Zymography on SDS-gelatin was used to determine gelatinase activity ${ }^{3}$ in sputum according to a method previously described. ${ }^{72}$ Each supernatant obtained from sputum was diluted (1:3) with PBS and $30 \mu \mathrm{l}$ of the sample was subjected to electrophoresis on $11 \%$ polyacrylamide gels containing $1 \mathrm{mg} / \mathrm{ml}$ gelatin in the presence of sodium dodecyl sulfate (SDS-PAGE) under non-reducing conditions. After electrophoresis, gels were washed in Triton X100 for 1 hour, rinsed briefly, and incubated at $37^{\circ} \mathrm{C}$ for 24 hours in buffer containing $100 \mathrm{mM}$ TRIS $\mathrm{HCl} \mathrm{pH} 7.40$ and $10 \mathrm{mM} \mathrm{CaCl}_{2}$. After incubation the gels were stained with Coomassie Brilliant Blue R250 and then destained in a solution of $7.5 \%$ acetic acid with 5\% methanol. Zones of enzymatic activity were indicated by negative staining: areas of proteolysis appeared as clear bands against a blue background.

\section{Measurement of MMP-9 and TIMP- 1}

The absolute values of MMP-9 and TIMP-1 levels in sputum were measured using enzyme immunoassay kits (Fuji Chemical Industries Ltd, Toyama, Japan). ${ }^{25}$ Assays were performed following the manufacturer's instructions. The MMP-9 assay detects pro (92 kDa) and intermediate forms of MMP-9 with $83 \mathrm{kDa}$ as well as their complexed forms with TIMP-1. The TIMP-1 assay detects free or complexed forms of TIMP-1. This kit does not crossreact with TIMP-2. The detection limits were $3.1 \mathrm{ng} / \mathrm{ml}$ for MMP-9 and $1.2 \mathrm{ng} / \mathrm{ml}$ for TIMP-1.

\section{CT scans and analysis of airway wall thickness}

CT scanning and analysis was performed as reported previously. ${ }^{19}{ }^{26}$ Briefly, using a Toshiba X-Vigor CT scanner (Toshiba, Tokyo, Japan), helical CT scanning was performed at $120 \mathrm{kVp}, 50 \mathrm{~mA}, 3 \mathrm{~mm}$ collimation, and pitch 1. Images were reconstructed using the FC 10 algorithm at $2 \mathrm{~mm}$ spacings. A targeted reconstruction of the right lung was performed using a subject-specific field of view (FOV) (153$214 \mathrm{~mm})$. These CT data were transferred to a Power PC personal computer via magneto-optical disk and analysed using custom software written in C programming language (Symantec C++, Symantec Corp, CA, USA). For the airway analysis, a cross section of the apical bronchus of the right upper lobe at its origin was selected by a consensus reading of two pulmonologists (HM and AN). The following airway parameters were then measured automatically on the computer: luminal area, short and long radii of the lumen, and absolute airway thickness $(\mathrm{T})$. Outer area of the bronchus (Ao), airway wall area (WA) and percentage wall area $(\mathrm{WA} \%$, WA/Ao $\times 100)$ were calculated, under the assumptions that the airway was a true circle in the cross sectional plane and that $\mathrm{T}$ was constant throughout the wall. Because airway size may be affected by body size, WA and T were normalised using body surface area (BSA). ${ }^{19}{ }^{26}$ Airway wall thickness was estimated as WA/BSA, WA\%, and T/ $\sqrt{B S A}$. A detailed description of these measures has been published previously. ${ }^{26}$

\section{Pulmonary function}

Using a Chestac-65V (Chest MI Corp, Tokyo, Japan), we measured $\mathrm{FEV}_{1}$, mid forced expiratory flow $\left(\mathrm{FEF}_{25-75 \%}\right)$, and maximum expiratory flow at the quartile of FVC $\left(\mathrm{MEF}_{25 \%}\right)$ 15 minutes after inhalation of $200 \mu \mathrm{g}$ salbutamol via metered dose inhaler to assess the degree of chronic airflow obstruction.

\section{Statistical analysis}

Stat View software (SAS Institute Inc, Cary, NC, USA) was used. The results are expressed as mean (SD). Relationships between data were analysed using Spearman's rank correlation test. $\mathrm{p}$ values of $<0.05$ were considered significant.

Table 1 Characteristics and outcomes of study patients $(n=26)$

\begin{tabular}{|c|c|}
\hline Age (years) & $53.8(16.3)$ \\
\hline $\operatorname{Sex}(F / M)$ & $11 / 15$ \\
\hline Disease duration (years) & $8.6(8.3)$ \\
\hline Disease severity (steps $1: 2: 3: 4$ )* & $1: 9: 13: 3$ \\
\hline Atopic status (yes/no)† & $16 / 10$ \\
\hline $\mathrm{FEV}_{1}(\%$ pred) $\ddagger$ & $95.8(17.2)$ \\
\hline $\mathrm{FEV}_{1}$ (\%pred)§ & $102.1(17.8)$ \\
\hline $\mathrm{FEV}_{1} / \mathrm{FVC}(\%) \S$ & $78.3(9.7)$ \\
\hline $\mathrm{FEF}_{25-75 \%}$ (\%pred)§ & $73.2(30.7)$ \\
\hline $\mathrm{MEF}_{25 \%}$ (\%pred)§ & $54.8(27.2)$ \\
\hline Inhaled steroid ( $\mu \mathrm{g} /$ day) & $816(270)$ \\
\hline WA $/$ BSA $\left(\mathrm{mm}^{2} / \mathrm{m}^{2}\right)$ & $17.9(3.8)$ \\
\hline WA\% $(\%)$ & $66.7(5.6)$ \\
\hline $\mathrm{T} / \sqrt{\mathrm{BSA}}(\mathrm{mm} / \mathrm{m})$ & $1.2(0.1)$ \\
\hline Sputum MMP-9 levels (ng/ml) & $598.2(388.6)$ \\
\hline Sputum TIMP-1 levels (ng/ml) & $855.0(497.3)$ \\
\hline Molar ratio of MMP-9/TIMP-1 & $0.22(0.13)$ \\
\hline \multicolumn{2}{|c|}{ 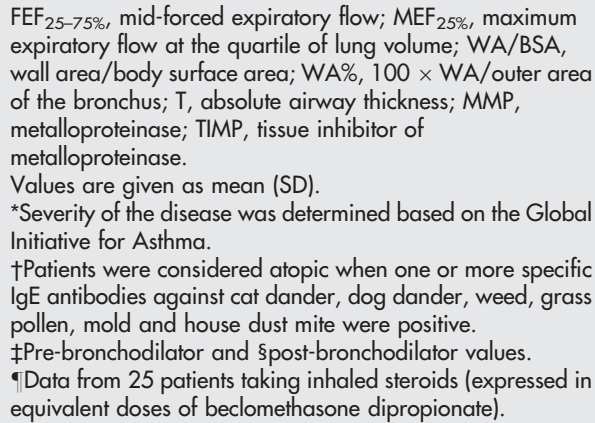 } \\
\hline
\end{tabular}


Table 2 Correlation coefficients ( $r$ values) between sputum markers and airway wall thickness or post-bronchodilator pulmonary function

\begin{tabular}{|c|c|c|c|}
\hline & MMP-9 levels & TIMP-1 levels & $\begin{array}{l}\text { Molar ratio of } \\
\text { MMP-9/TIMP-1 }\end{array}$ \\
\hline WA/BSA $\left(\mathrm{mm}^{2} / \mathrm{m}^{2}\right)$ & 0.24 & $0.42^{*}$ & -0.12 \\
\hline WA\% & $-0.47 \dagger$ & 0.15 & $-0.64 \ddagger$ \\
\hline $\mathrm{T} / \mathrm{BSA}(\mathrm{mm} / \mathrm{m})$ & -0.15 & $0.41^{*}$ & $-0.54 \S$ \\
\hline $\mathrm{FEV}_{1}$ (\%pred) & 0.24 & 0.12 & 0.25 \\
\hline $\mathrm{FEF}_{25-75 \%}$ (\%pred) & 0.11 & -0.26 & $0.41^{*}$ \\
\hline $\mathrm{MEF}_{25 \%}$ (\%pred) & 0.20 & -0.21 & $0.42^{*}$ \\
\hline
\end{tabular}

\section{RESULTS}

The clinical characteristics and outcomes of the 26 patients are shown in table 1 . One patient regarded as of step 1 severity was treated only with a short acting inhaled $\beta$ agonist as required. Others were treated with inhaled corticosteroids for at least 3 months before the study. Two of the 26 patients were examined for MMP-9 or TIMP-1 levels alone because the sputum samples of these patients were too small to analyse both. As reported previously, ${ }^{6} 25$ zymographic analysis of sputum samples from the patients showed a major band of enzymatic activity at $92 \mathrm{kDa}$ which corresponds to pro-MMP-9 (data not shown).

Table 2 shows correlation coefficients between the sputum indices and the measures of airway wall thickness or airflow obstruction. Sputum MMP-9 levels were inversely correlated with WA\% and TIMP-1 levels were positively correlated with

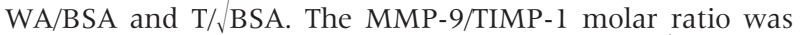
inversely correlated with WA\% (fig 1 ) and $\mathrm{T} / \sqrt{\mathrm{BSA}}$ and positively correlated with post-bronchodilator values of $\mathrm{FEF}_{25-75 \%}$ and $\mathrm{MEF}_{25 \%}$ but not with $\mathrm{FEV}_{1}$ (table 2). No significant correlation was observed between disease duration or severity and the sputum indices (data not shown).

The MMP-9 levels were significantly correlated with the absolute number of sputum neutrophils $(r=0.47, \mathrm{p}=0.036)$ and marginally with that of macrophages $(r=0.43$, $\mathrm{p}=0.058)$. The TIMP-1 levels tended to correlate with the absolute number of neutrophils $(r=0.38, \mathrm{p}=0.099)$. No significant correlation was found between sputum eosinophil number and either MMP-9 or TIMP-1 levels (data not shown).

\section{DISCUSSION}

This is the first study to evaluate the relationship between airway wall thickness (assessed by CT scanning) and sputum levels of MMP-9, TIMP-1, and their molar ratio in asthmatic patients. We have shown that an absolute increase in TIMP-1 or its relative excess over MMP-9 in the asthmatic airway

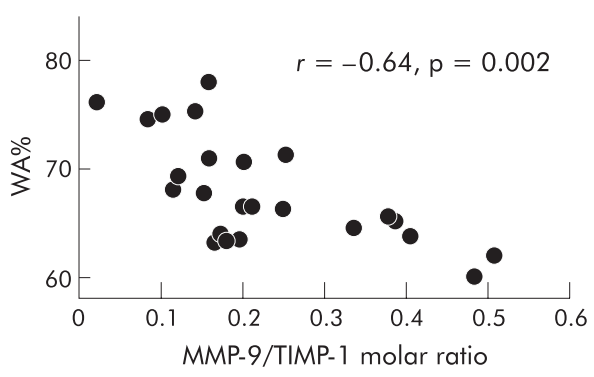

Figure 1 Correlation between WA\% $(100 \times$ wall area/outer area of the bronchus) and MMP-9/TIMP-1 molar ratio. may be associated with airway wall thickening, as well as with chronic airflow obstruction.

Vignola and colleagues have shown that the molar ratio between MMP-9 and TIMP-1 in the sputum of asthmatic patients is positively correlated with $\mathrm{FEV}_{1}$. The patients had not received any form of corticosteroids during the 2 months before the study. The authors indicated that the excess of TIMP-1 may lead to airflow obstruction, possibly by a role in the pathogenesis of ECM remodelling. ${ }^{6}$ Bossé and colleagues have evaluated the relationship between the responsiveness of $\mathrm{FEV}_{1}$ to oral corticosteroid treatment (methylprednisolone $40 \mathrm{mg} /$ day for 2 weeks) and the pretreatment serum molar ratio of MMP-9/TIMP-1 in severe asthmatic patients requiring high doses of inhaled corticosteroids. ${ }^{17}$ The baseline serum MMP-9/TIMP-1 ratio strongly correlated with the degree of increase in $\mathrm{FEV}_{1}$ after oral corticosteroid treatment. The authors concluded that the excess of TIMP-1 may lead to irreversible airflow obstruction, possibly through airway fibrosis. Our findings that the sputum MMP-9/TIMP-1 ratio correlated with $\mathrm{FEF}_{25-75 \%}$ and $\mathrm{MEF}_{25 \%}$ are consistent with those of Vignola et $a l^{6}$ and Bossé et $a l,{ }^{17}$ both showing an association between the excess TIMP-1 and airflow obstruction. Unlike these studies, we used post-bronchodilator values of pulmonary function which are more indicative of "irreversible" functional changes. We also used sputum samples which provide a more direct measure of airway pathophysiology than serum samples as used by Bossé et al. ${ }^{17}$ The reason why the molar ratio was only associated with $\mathrm{FEF}_{25-75 \%}$ and $\mathrm{MEF}_{25 \%}$ but not with $\mathrm{FEV}_{1}$ in our study may be that a fixed airflow obstruction was limited to the peripheral airways only because our patients may have had milder disease than those in the previous studies. ${ }^{6}{ }^{17}$ Correlations between the sputum indices and peripheral airflow obstruction are relevant because induced sputum is considered to originate not only from the central but also from the small airways and alveoli. ${ }^{27}$

We have shown, for the first time to our knowledge, an association between excess TIMP-1 and whole airway wall thickening. Specifically, the sputum TIMP-1 levels were positively correlated with-and the molar ratio of MMP-9/ TIMP-1 inversely correlated with-airway wall thickness as assessed by CT scanning. Although CT scanning cannot identify the pathological details that may contribute to airway wall thickening, ECM deposition in the airways through excess TIMP-1 probably contributed to the observed thickening. A recent study of a murine model of asthma employing chronic ovalbumin challenge shows that the TIMP-1 level in BAL fluid is increased after 12 weeks of repeated antigen challenge and that this increase is sustained during the following 20 weeks with the same treatment. In parallel, excessive collagen deposition in the lung emerges at 16 weeks of treatment and persists thereafter. The authors 
deduce a possible association between the increase in TIMP-1 and ECM deposition in their model. ${ }^{28}$ In the airways of patients with asthma, ECM deposition is seen not only in the SBM but also around smooth muscle cells and possibly in the adventitial layer, ${ }^{2}$ which may gather volume and contribute to the whole airway wall thickening. In fact, the degree of SBM thickening correlates with the degree of airway wall thickening as assessed histologically ${ }^{29}$ and by CT scanning. ${ }^{22}$ TIMP-1 excess may therefore be associated with ECM deposition and consequent whole airway wall thickening. The latter may be related to chronic airway obstruction as suggested by previous CT studies. ${ }^{19-22}$

In our study, sputum MMP-9 levels correlated significantly with the number of neutrophils and marginally with the number of macrophages. Our results are consistent with those of previous human ${ }^{6}{ }^{10}$ and murine studies. ${ }^{28}$ These cells might therefore be potential sources of MMP-9 in the asthmatic airways, although eosinophils are also considered as candidate cells by other investigators. ${ }^{30}$

Uncontrolled airway inflammation or uncontrolled disease status may potentially affect airway wall thickness. All 26 patients in our study had experienced full symptom control for at least 1 month, and all but one had been treated with inhaled corticosteroids for at least 3 months. We selected these patients for our study to reduce the effect of inflammation as much as possible in order to better address the "irreversible" component of airway wall thickening using CT. ${ }^{21}{ }^{22}$ Although the levels of sputum indices might or might not have been modified by corticosteroid treatment, ${ }^{31-33}$ the association we found between the sputum indices and pulmonary function is consistent with those of a previous study ${ }^{6}$ conducted in patients not treated with corticosteroids.

The three measures of airway wall thickness used in our study are distinct from each other. WA/BSA may not be affected by bronchoconstriction, unlike WA\% or $\mathrm{T} / \sqrt{\mathrm{BSA}}$; WA $\%$ is a corrected index of airway wall thickness by airway size, whereas $\mathrm{T} / \sqrt{\mathrm{BSA}}$ indicates an absolute thickness of the wall. ${ }^{34}$

One possible limitation of our study is that sputum levels of MMP-9 and TIMP-1 might not reflect the actual behaviour of the enzymes within bronchial tissue. However, both mucosal expression ${ }^{35}$ and sputum levels ${ }^{6}$ of MMP-9 as well as TIMP-1 are consistently increased in patients with asthma compared with healthy controls. Increases in TIMP-1 relative to MMP-9 in induced sputum ${ }^{33}$ and increased expression of TIMP-1 in bronchial tissue ${ }^{31}$ are consistently observed following systemic or inhaled corticosteroid treatment. It is therefore reasonable to assume that sputum levels of MMP-9 and TIMP-1 may indirectly reflect their levels in the airway mucosa.

In conclusion, the data from the present study suggest the involvement of excess TIMP-1 in the development of airway wall thickening and chronic airflow obstruction in asthmatic patients. The regulatory mechanisms responsible for the balance between MMP-9 and TIMP-1, and its possible pharmacological modulation, need to be addressed in future studies.

\section{ACKNOWLEDGEMENTS}

The authors thank Ryuzo Tanaka, Goro Saji, Hiroyuki Akazawa, Noboru Narai and Miho Morimoto for their radiological technical support.

\footnotetext{
Authors' affiliations

H Matsumoto, A Niimi, M Takemura, T Ueda, M Minakuchi, R Tabuena, T Mio, Y Ito, S Muro, T Hirai, M Mishima, Department of Respiratory Medicine, Kyoto University Graduate School of Medicine, Kyoto, Japan K Chin, Department of Physiotherapy, Kyoto University Graduate School of Medicine, Kyoto, Japan
}

S Morita, S Fukuhara, Department of Epidemiology and Health Care Research, Kyoto University Graduate School of Medicine, Kyoto, Japan M Minakuchi, Department of Respiratory Medicine, Tenri Hospital, Nara, Japan

Supported by AstraZeneca Asthma Research Award 2000 (Japanese Society of Allergology and Japan Allergy Foundation).

The authors have no competing interests.

\section{REFERENCES}

1 Jeffery PK. Remodeling in asthma and chronic obstructive lung disease. Am J Respir Crit Care Med 2001;164:S28-38.

2 Bai TR, Cooper J, Koelmeyer T, et al. The effect of age and duration of disease on airway structure in fatal asthma. Am J Respir Crit Care Med 2000; 162:663-9

3 Woessner FJ Jr. Matrix metalloproteinases and their inhibitors in connective tissue remodeling. FASEB J 1991;5:2145-54.

4 Gruber BL, Sorbi D, French DL, et al. Markedly elevated serum MMP-9 (gelatinase-B) levels in rheumatoid arthritis: a potentially useful laboratory marker. Clin Immunol Immunopathol 1996;78:161-71.

5 Segura VL, Pardo A, Gaxiola M, et al. Upregulation of gelatinases A and B, collagenases 1 and 2, and increased parenchymal cell death in COPD. Chest 2000; 117:684-94.

6 Vignola AM, Riccobono L, Mirabella A, et al. Sputum metalloproteinase-9/ tissue inhibitor of metalloproteinase- 1 ratio correlates with airflow obstruction in asthma and chronic bronchitis. Am J Respir Crit Care Med 1998; 158:1945-50

7 Mautino G, Oliver N, Chanez $P$, et al. Increased release of matrix metalloproteinase-9 in bronchoalveolar lavage fluid and by alveolar macrophages of asthmatics. Am J Respir Cell Mol Biol 1997; 17:583-91.

8 Oshita Y, Koga T, Kamimura T, et al. Increased circulating 92 kDa matrix metalloproteinase (MMP-9) activity in exacerbations of asthma. Thorax 2003;58:757-60.

9 Kelly EAB, Busse WW, Jarjour NN. Increased matrix metalloproteinase-9 in the airway after allergen challenge. Am J Respir Crit Care Med 2000;162:1157-61

10 Wenzel SE, Balzar S, Cundall M, et al. Subepithelial basement membrane immunoreactivity for matrix metalloproteinase-9: association with asthma severity, neutrophilic inflammation, and wound repair. J Allergy Clin Immunol 2003;111:1345-52.

11 Okada S, Kita H, George TJ, et al. Migration of eosinophils through basement membrane components in vitro: role of matrix metalloproteinase-9. Am J Respir Cell Mol Biol 1997; 17:519-28.

12 Nagase H. Matrix metalloproteinases. In: Hopper NM, eds. Zinc metalloproteinases in health and diseases. London: Taylor and Francis, 1996: 153-204

13 Hayakawa T. Tissue inhibitors of metalloproteinase and their cell growthpromoting activity. Cell Struct Funct 1994;19:109-14.

14 Kikuchi K, Kubo M, Sato S, et al. Serum tissue inhibitor of metalloproteinases in patients with systemic sclerosis. J Am Acad Dermatol 1995;33:973-8.

15 Selman M, Ruiz V, Cabrera S, et al. TIMP-1, -2, -3, and -4 in idiopathic pulmonary fibrosis. A prevailing nondegradative lung microenvironment? Am J Physiol Lung Cell Mol Physiol 2000;279:L562-74.

16 Mautino G, Henriquet C, Jaffuel D, et al. Tissue inhibitor of metalloproteinase1 levels in bronchoalveolar lavage fluid from asthmatic subjects. Am J Respir Crit Care Med 1999;160:324-30.

17 Bossé M, Chakir J, Rouabhia M, et al. Serum matrix metalloproteinase-9: tissue inhibitor of metalloproteinase-1 ratio correlates with steroid responsiveness in moderate to severe asthma. Am J Respir Crit Care Med 1999; 159:596-602.

18 McParland PE, Macklem PT, Pare PD. Airway wall remodelling: friend or foe? J Appl Physiol 2003:95:426-34

19 Niimi A, Matsumoto H, Amitani R, et al. Airway wall thickness in asthma assessed by computed tomography: relation to clinical indices. Am J Respir Crit Care Med 2000;162:1518-23.

20 Niimi A, Matsumoto H, Amitani R, et al. Effect of short-term treatment with inhaled corticosteroid on airway wall thickening in asthma. Am J Med 2004; 1 16:725-31.

21 Little SA, Sproule MW, Cowan MD, et al. High resolution computed tomographic assessment of airway wall thickness in chronic asthma: reproducibility and relationship with lung function and severity. Thorax 2002; 57:247-53.

22 Kasahara K, Shiba K, Ozawa T, et al. Correlation between the bronchial subepithelial layer and whole airway wall thickness in patients with asthma. Thorax 2002;57:242-6.

23 Takishima T, Hida W, Sasaki $\mathrm{H}$, et al. Direct-writing recorder of the doseresponse curves of the airway to methacholine. Chest 1981;80:600-6.

24 Pin I, Gibson PG, Kolendowicz R, et al. Use of induced sputum cell counts to investigate airway inflammation in asthma. Thorax 1992;47:25-9.

25 Takemura $M$, Niimi $A$, Minakuchi $M$, et al. Bronchial dilatation in asthma: relation to clinical and sputum indices. Chest 2004;125:1352-8.

26 Nakano Y, Muro S, Sakai H, et al. Computed tomographic measurements of airway dimensions and emphysema in smokers. Correlation with lung function. Am J Respir Crit Care Med 2000;162:1102-8.

27 Gershman NH, Liu $\mathrm{H}$, Wong HH, et al. Fractional analysis of sequential induced sputum samples during sputum induction: evidence that different lung compartments are sampled at different time points. J Allergy Clin Immunol 1999; 104:322-8 
28 Corbel M, C-Maugendre S, Germain N, et al. Enhancement of gelatinase activity during development of subepithelial fibrosis in a murine model of asthma. Clin Exp Allergy 2003;33:696-704.

29 James AL, Maxwell PS, Pearce-Pinto G, et al. The relationship of reticular basement membrane thickness to airway wall remodeling in asthma. Am J Respir Crit Care Med 2002;166:1590-5.

30 Ohno I, Ohtani $\mathrm{H}, \mathrm{Nitta} Y$, et al. Eosinophils as a source of matrix metalloproteinase-9 in asthmatic airway inflammation. Am J Respir Cell Mol Biol 1997; 16:212-9.

31 Hoshino M, Takahashi M, Takai Y, et al. Inhaled corticosteroids decrease subepithelial collagen deposition by modulation of the balance between matrix metalloproteinase- 9 and tissue inhibitor of metalloproteinase-1 expression in asthma. J Allergy Clin Immunol 1999;104:356-63.
32 Cundall $M$, Sun $Y$, Miranda $C$, et al. Neutrophil-derived matirx metalloporteinase-9 is increased in severe asthma and poorly inhibited by glucocorticoids. J Allergy Clin Immunol 2003;1 12:1064-71

33 Tanaka H, Miyazaki N, Oashi K, et al. Sputum matrix metalloproteinase9:tissue inhibitor of metalloproteinase-1 ratio in acute asthma. J Allergy Clin Immunol 2000; 105:900-5.

34 King GG, Müller NL, Paré PD, et al. Evaluation of airways in obstructive pulmonary disease using high-resolution computed tomography. Am J Respir Crit Care Med 1999:159:992-1004.

35 Hoshino M, Nakamura Y, Sim JJ, et al. Bronchial subepithelial fibrosis and expression of matrix metalloproteinase-9 in asthmatic airway inflammation. $\mathrm{J}$ Allergy Clin Immunol 1998;102:703-8.

\section{LUNG ALERT}

Absence of C/EBPa may cause bronchial smooth muscle proliferation in asthma

$\Delta$ Roth $\mathrm{M}$, Johnson PRA, Borger $\mathrm{P}$, et al. Dysfunctional interaction of $\mathrm{C} / \mathrm{EBP} \alpha$ and the glucocorticoid receptor in asthmatic bronchial smooth muscle cells. N Engl J Med 2004;351:560-74

$P$

roliferation of bronchial smooth muscle (BSM) cells occurs in the airways of asthmatic patients. Glucocorticoids exert antiproliferative effects via CCAAT/enhancer binding protein $\alpha(\mathrm{C} / \mathrm{EBP} \alpha)$, an antiproliferative transcription factor. This paper studies signalling pathways in BSM cell proliferation from 20 asthmatic and 26 non-asthmatic subjects (lung cancer/CF/emphysema).

A series of elegant experiments (using immunoblotting/electrophoresis) first established that, in response to glucocorticoid, asthmatic and non-asthmatic BSM cells display similar glucocorticoid receptor (GR) characteristics, similar anti-inflammatory effects (interleukin (IL)-6 suppression), and a similar reduction in IL-6 suppression with a GR inhibitor. However, whereas glucocorticoid significantly suppresses cell proliferation in control BSM cells (by 24-28\%), no such suppression is seen in asthmatic BSM cells. None of the asthmatic cell lines expressed the $\mathrm{C} / \mathrm{EBP} \alpha$ transcription factor whereas all controls did (tissue specific to BSM). Transfection of asthmatic cells with a C/EBP $\alpha$ expression vector significantly slowed (and allowed steroids to suppress) proliferation.

The authors conclude that asthmatic BSM cell proliferation results from absence of C/ $\mathrm{EBP} \alpha$, which also explains the lack of steroid suppression. Steroid treatment suppresses IL- 6 production in both groups, therefore demonstrating two distinct signalling pathways: antiinflammatory (cytokine mediated) and antiproliferative (transcription factor mediated).

Why asthmatic BSM cells lack C/EBP $\alpha$ remains unclear (previous steroid use and treatment length were often unknown, perhaps influencing in vitro response) and whether CF or emphysema subjects can be used as controls is debatable. The implication that steroids do not suppress BSM proliferation in asthma is clearly important and the demonstration of two distinct signalling pathways merits further investigation.

N M Rahman

Specialist Registrar, Respiratory Medicine, Horton Hospital, Banbury, Oxon, UK; nai_rahman@yahoo.co.uk 